\title{
Visualização dos dados de candidatos a auxílios socioeconômicos em grafos: estudo de caso no Instituto Federal de Minas Gerais (IFMG)
}

\section{Visualization of candidate data for socioeconomic aid in graphs: case study at the Federal Institute of Minas Gerais (IFMG)}

\author{
Eduardo Cardoso Melo ${ }^{1}$, Marcello Peixoto Bax², Yuri Bento Marques ${ }^{3}$ \\ ${ }^{1}$ Instituto Federal do Norte de Minas Gerais (IFNMG), Belo Horizonte, MG, Brasil. ORCID: https://orcid.org/0000-0002-1323-5859 \\ 2 Universidade Federal de Minas Gerais (UFMG), Belo Horizonte, MG, Brasil. ORCID: https://orcid.org/0000-0003-0503-3031 \\ ${ }^{3}$ Instituto Federal do Norte de Minas Gerais (IFNMG), Belo Horizonte, MG, Brasil. ORCID: https://orcid.org/0000-0003-3414-2325
}

Autor para correspondência/Mail to: Eduardo Cardoso Melo, eduardo.melo@ifmg.edu.br

Recebido/Submitted: 25 de agosto de 2021; Aceito/Approved: 1 de dezembro de 2021

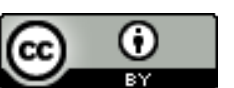

Copyright (C) 2022 Melo, Bax, \& Marques. Todo o conteúdo da Revista (incluindo-se instruções, política editorial e modelos) está sob uma licença Creative Commons Atribuição 4.0 Internacional. Ao serem publicados por esta Revista, os artigos são de livre uso em ambientes educacionais, de pesquisa e não comerciais, com atribuição de autoria obrigatória. Mais informações em http://revistas.ufpr.br/atoz/about/submissions\#copyrightNotice.

\begin{abstract}
Resumo
Introdução: a expansão da rede federal de ensino superior brasileira nas últimas duas décadas foi possível, em grande parte, graças à implementação de ações governamentais como o Programa Nacional de Assistência Estudantil (PNAES). Nele, os recursos orçamentários são descentralizados para as instituições de ensino que, por sua vez, ficam responsáveis por selecionar aqueles candidatos que apresentam maior demanda para recebimento de auxílios socioeconômicos. Este artigo propõe uma abordagem diferenciada para visualizar as relações entre os dados de tais candidatos, aprimorando o processo de análise conduzido por Assistentes Sociais. Método: foi realizado um estudo de caso no Instituto Federal de Minas Gerais (IFMG) para demonstrar a viabilidade de implementação e uso da solução proposta a partir de dados reais da instituição, coletados diretamente da base de candidatos a auxílios socioeconômicos. Resultados: a apresentação dos dados e dos relacionamentos entre as entidades podem ser melhor visualizados pelos Assistentes Sociais e contribuir diretamente para entender a real situação dos candidatos. Conclusão: a análise das informações dos candidatos pode se beneficiar da construção de conhecimentos a partir de dados organizados em grafos, em vez de representações textuais.
\end{abstract}

Palavras-chave: Visualização em Grafos; Gestão da Informação; Assistência Estudantil.

\begin{abstract}
Introduction: the expansion of the Brazilian federal higher education network in the last two decades was made possible, in large part, thanks to the implementation of governmental actions such as the National Student Assistance Program (PNAES). In it, budgetary resources are decentralized to educational institutions, which, in turn, are responsible for selecting those candidates who have the greatest demand for receiving socio-economic assistance. This paper proposes a differentiated approach to visualize the relationships between the data of such candidates, improving the analysis process conducted by Social Assistants. Method: a case study was carried out at the Federal Institute of Minas Gerais (IFMG) to demonstrate the feasibility of implementing and using the proposed solution based on real data from the institution, collected directly from the base of applicants for socioeconomic assistance. Results: the presentation of data and relationships between entities can be better viewed by Social Workers and contribute directly to understanding the real situation of the candidates. Conclusions: analyzis of candidate information can benefit from the ability to build knowledge based on the visualization of data in graphs compared to that based on textual reports.
\end{abstract}

Keywords: Graph Visualization; Information Management; Student Assistance.

\section{INTRODUÇÃO}

A educação superior no Brasil passou por grandes transformações nas últimas duas décadas, tanto em termos de oferta de cursos e vagas, quanto em relação ao perfil dos alunos. Se antes as universidades e faculdades ficavam localizadas em grandes centros, obrigando os estudantes a se mudarem para seguir com os estudos, a expansão do ensino privado, e também do público, levou a uma interiorização das instituições de ensino superior (IES) e, consequentemente, criou novas possibilidades para pessoas que não teriam condições de arcar com todos os custos que envolvem esta mudança (Camargo \& Araújo, 2018). No setor público, o Programa de Reestruturação e Expansão das Universidades Federais (Reuni) é considerado um dos principais responsáveis pelo crescimento do número de Instituições Federais de Ensino Superior (IFES), campi, cursos e vagas no Brasil. A proposta era fornecer condições para o estabelecimento de unidades educacionais em regiões até então não cobertas pela rede federal, seja pela criação de universidades ou pela abertura de novos campi de instituições já existentes. Entre 2003 e 2011 houve um crescimento de aproximadamente $111 \%$ no número de vagas ofertadas em universidades federais (de 109.184 para 231.530), além de quase $100 \%$ de incremento do número de alunos matriculados, passando de 596.219 para 1.029.141 (Ministério da Educação, 2012).

A concepção dos Institutos Federais de Ensino (IFES) ocorreu neste contexto de valorização do ensino público, aumentando a capilaridade e alcance da rede federal de educação profissional para os mais remotos locais do 
país por meio de mais 660 unidades vinculadas a quase 40 Institutos Federais (Ministério da Educação [MEC], 2021). Entretanto, o aumento da oferta de cursos e vagas fez surgir outras questões relacionadas à gestão das IFES, em especial sobre a permanência dos alunos na instituição. Ao possibilitar o ingresso de indivíduos com variados perfis e realidades de vida, a expansão da rede federal fez com que novas variáveis tivessem influência na continuidade dos estudos, tais como renda mensal familiar per capita, condições de moradia e transporte até o campus, recursos para aquisição de alimentos e materiais escolares, dentre outras. A pesquisa realizada pela Associação Nacional dos Dirigentes das Instituições Federais de Ensino Superior (Associação Nacional dos Dirigentes das Instituições Federais de Ensino Superior [ANDIFES, 2019) apresenta um perfil variado do atual estudante de graduação no país, o que sugere a necessidade de adaptação da gestão pública para um entendimento mais profundo dos fatores que impactam na permanência dos ou evasão dos estudantes. Uma das ações realizadas pelas IFES com o objetivo de manter os discentes no ambiente acadêmico foi o incremento na oferta de programas de assistência estudantil para os mais vulneráveis, tanto econômica quanto socialmente. $\mathrm{O}$ apoio de tais programas seria uma estratégia eficaz para a permanência discente. Contudo, a assistência estudantil não era uma realidade em todas as instituições públicas na década de 2000, variando muito em função do tamanho, orçamento e estrutura organizacional de cada universidade (E. H. M. Santos, 2020).

Visando a fortalecer, em toda a rede federal, as políticas de assistência estudantil, bem como oferecer condições para que os estudantes permaneçam na instituição até a conclusão do curso, o Governo Federal criou, em 2007, o Programa Nacional de Assistência Estudantil (PNAES). Em 2010 o Programa foi institucionalizado e publicado como Decreto. O PNAES indicava a necessidade de atenção para com moradia, alimentação, transporte e apoio pedagógico aos alunos (Decreto n. 7.234, 2010). Desde então, conforme apresenta E. H. M. Santos (2020), as IFES passaram a contar com uma orientação formal que fomentou a assistência estudantil com dotação orçamentária específica. O PNAES requer a realização de processos seletivos com critérios e metodologias para a definição de quais discentes participarão dos programas assistenciais. Além do atendimento a tais critérios, os candidatos devem ter renda familiar per capita de até um salário mínimo e meio. Uma forma de coletar os dados dos interessados é o preenchimento de questionários socioeconômicos e a entrega de documentação comprobatória. A consolidação desses dados permite realizar uma análise mais detalhada da realidade de cada aluno e subsidiar a tomada de decisão para atribuir ou não determinado auxílio. Contudo, dependendo da amplitude da coleta de dados, da estrutura organizacional da IFES e da disponibilidade de recursos computacionais, a análise pode ficar prejudicada e resultar na não atribuição de auxílios para aqueles mais necessitados. Reis e Gonçalves (2018) mencionam que o Instituto Federal de Brasília define os alunos contemplados a partir da análise de quinze indicadores, já o Instituto Federal de Minas Gerais considera dez indicadores (Instituto Federal de Minas Gerais [IFMG], 2020).

Em termos práticos, o volume de dados coletados pode ser tamanho que o efeito é justamente o contrário ao desejado, isto é, ao invés de servir de base para uma decisão criteriosa, acaba gerando trabalho extra até que se consiga chegar a uma interpretação mínima de todas as informações obtidas. Os recursos financeiros direcionados à assistência estudantil não conseguem atender a totalidade de interessados em muitas IFES do país (L. B. Silva \& Costa, 2018), o que indica a necessidade de identificar com exatidão os discentes em situação de vulnerabilidade. Além do volume dos dados, existe o fato de que muitos deles são armazenados em formatos que não colaboram para o entendimento humano, ou demandam tratamento extra para serem lidos, dificultando o processo de análise da realidade de cada aluno.

Consideradas as dificuldades para a análise dos dados, o presente trabalho buscou aplicar recursos de visualização da informação em grafos com vistas a proporcionar melhor entendimento sobre os dados de candidatos a auxílios socioeconômicos, em especial no que se refere à identificação de relacionamentos que não poderiam ser encontrados facilmente por meio de análises em documentos textuais. A aplicabilidade de tal estudo se justifica sob variados aspectos. O primeiro é que uma análise bem fundamentada dos dados de cada candidato pode fazer com que os recursos sejam direcionados para alunos que precisam do auxílio para a continuidade dos seus estudos. Outro fator a ser considerado é o montante despendido pelo Governo Federal com o PNAES, tendo alcançado quase 900 milhões de reais efetivamente pagos em 2019, conforme Moraes e Spessatto (2020). Mesmo sendo um alto valor absoluto, quando dividido pelas diversas ações assistenciais das IFES e entre o grande número de alunos interessados, nem todos que necessitam do auxílio são contemplados e, eventualmente, podem não permanecer na instituição.

A principal contribuição deste trabalho consiste na introdução de uma abordagem complementar à análise tradicional dos dados de candidatos a auxílios socioeconômicos, disponibilizando aos tomadores de decisão recursos que promovam uma visão integrada (e não fragmentada) das diversas entidades envolvidas no processo de análise de candidatos a auxílios socioeconômicos em IFES. O artigo foi estruturado com esta introdução aos assuntos envolvidos; em seguida, uma seção destinada a conceituar os principais temas e abordar trabalhos relacionados; outra seção para caracterizar a metodologia; uma seção que apresenta e discute os resultados obtidos no estudo; uma seção com as considerações finais dos autores e, por fim, as referências bibliográficas que embasaram o estudo. 


\section{FUNDAMENTAÇÃO TEÓRICA E TRABALHOS RELACIONADOS}

\section{Plano Nacional de Assistência Estudantil (PNAES)}

O PNAES foi instituído, inicialmente, por meio da Portaria Normativa $n^{\circ} 39$ de 12/12/2007 e formalizado como programa governamental com a publicação do Decreto no 7.234 de 19/08/2010. Seu objetivo principal é fomentar condições para que os estudantes das IFES brasileiras permaneçam matriculados e concluam seus cursos, contando com auxílios (geralmente financeiros) para o custeio de alimentação, moradia, transporte, saúde, inclusão digital, dentre outras áreas definidas pelo Decreto (Decreto n. 7.234, de 19 de julho de 2010, 2010). C. Santos, Abrantes, e Zonta (2021) ressaltam que, assim, a assistência estudantil passou a ter sua importância ampliada, servindo de estratégia para o enfrentamento das desigualdades regionais e sociais. Diversos estudos, dentre eles os de Moraes e Spessatto (2020), Andrade e Teixeira (2017) e Saccaro, França, e Jacinto (2016) indicam que os programas desenvolvidos pelas IFES no contexto do PNAES vêm sendo efetivos no auxílio à permanência dos alunos assistidos em seus estudos.

A assistência estudantil não apenas promove a entrada do aluno na instituição, mas também a sua retenção. Machado, Oliveira, e Freitas (2017), Eloi, Silva, Silveira, e Baeta (2019) e Braga (2017), buscaram analisar eventuais relações entre a oferta de programas assistenciais baseados no PNAES e o desempenho acadêmico dos alunos assistidos. Os resultados indicam que existe um impacto positivo entre o recebimento de benefícios como auxílios financeiros, moradia, alimentação e transporte, e o coeficiente de rendimento acadêmico dos alunos.

Sales (2018) e Martins, Araújo Júnior, e Rodrigues (2019) abordam o fato de que a distribuição de recursos orçamentários específicos para o PNAES fez com que as IFES ampliassem a oferta de programas de auxílio ao estudante para além daqueles feitos anteriormente com recursos próprios. Além disso, o PNAES constituiu-se em um marco legal para que as instituições construíssem suas próprias Políticas de Assistência Estudantil, conforme abordam Martins, Araújo Júnior, e Rodrigues (2018) e Silva Júnior (2020).

L. B. Silva e Costa (2018) exploraram o descompasso existente entre o aumento das possibilidades de acesso ao ensino superior público promovidas pelo PNAES e por outras políticas governamentais de expansão, e a capacidade das IFES atenderem todos os novos discentes com demandas socioeconômicas. Conforme apresentado em Associação Nacional dos Dirigentes das Instituições Federais de Ensino Superior [ANDIFES (2019) e Moraes e Spessatto (2020), enquanto a evolução do orçamento do PNAES desde 2015 é pequena, as instituições continuam ofertando o mesmo número de cursos e vagas, o que faz com que alunos não sejam atendidos, ou que o auxílio oferecido seja insuficiente de acordo com o mínimo necessário para a permanência na instituição.

\section{Gestão da Informação}

A sociedade moderna produz uma enorme quantidade de dados e necessita que eles sejam transformados em informações para que possam ter utilidade em processos decisórios. Quando esta situação é analisada do ponto de vista organizacional, no qual as decisões são capazes de afetar direta ou indiretamente um grande número de pessoas, ter uma gestão consistente dos dados e informações é fundamental para que os resultados sejam satisfatórios (T. E. Silva \& Tomaél, 2007).

Neste contexto, a Gestão da Informação é uma área que atua no gerenciamento dos diversos sistemas e processos existentes nas organizações utilizados em tarefas que envolvem dados e informações, tais como criação, aquisição, organização, armazenamento, distribuição e utilização. Seu objetivo é auxiliar as pessoas a acessarem informações úteis para diversos fins, especialmente aqueles relacionados com etapas de processos decisórios (Detlor, 2010). Outra atribuição da Gestão da Informação é fornecer meios para que seja possível a integração dos dados de diferentes sistemas. T. E. Silva e Tomaél (2016) reforçam este entendimento ao tratarem as novas demandas por sistemas que tenham habilidades de integração como exigência, sob pena de ser criado um ambiente no qual cada sistema resolve problemas específicos e opera de forma isolada. Em um mundo amplamente dependente de recursos tecnológicos, a todo momento coletando e intercambiando dados, a Gestão da Informação mostra-se fundamental.

No caso dos setores de assistência estudantil das IFES, não são muitos os trabalhos científicos que tratam do emprego de sistemas computacionais como meio para realização das tarefas administrativas. Melo, Silva, e Silva (2021), Chaves, Farias, Nunes, e Araújo (2021), Mariano (2020) e Terenciano, Salião, e Pedro (2018) relatam experiências válidas, nas quais tanto a Administração Pública quanto as pessoas envolvidas com processos de assistência estudantil passaram a ter mais acesso a informações úteis para a tomada de decisão a partir da utilização de sistemas computacionais.

\section{Análise baseada em grafos}

Um grafo é uma estrutura composta por conjuntos finitos de elementos nomeados como vértices e arestas, representados em formato de diagrama (Lucchesi, 1979). Os grafos podem ser classificados como orientados: quando seus vértices são direcionados para outro vértice em um único sentido, ou não orientados: quando as 
arestas possuem dupla direção (Szwrcfiter, 1984). Diversas situações podem ser representadas através desses diagramas, provendo uma visão mais conveniente das relações entre os seus pontos. A vinculação entre os nós existentes na rede mundial de computadores, os amigos de um indivíduo em determinada rede social, os componentes de uma malha elétrica e o melhor trajeto a ser percorrido por um veículo são exemplos que validam a importância das aplicações baseadas em grafos (Boaventura Netto \& Jurkiewicz, 2017).

O processo decisório também pode se beneficiar de análises advindas de um diagrama com grafos. Alguns autores desenvolveram propostas baseadas na Teoria dos Grafos para apoiar o processo de tomada de decisão em diferentes cenários, tais como Carvalho e Francisco (2017) para redução do viés cognitivo no processo de insight, (Bezerra, Medeiros, \& Oliveira, 2016) para monitorar postagens sobre Zika vírus e Sanz e Lopez (2018) para detectar ameaças em redes de computadores.

A visualização das informações no formato de grafos também pode atender a necessidades organizacionais específicas, especialmente quando o desafio é o grande volume de dados textuais, pois a representação visual dos relacionamentos entre os elementos permite abstrair entendimentos de forma mais direta e rápida. Neste sentido, destacam-se os trabalhos de Bezerra, Medeiros, e Oliveira (2014) para visualizar informações com opiniões sobre restaurantes no formato de grafos, Ramos (2016) para analisar trilhas de aprendizagem e Costa (2018) para visualizar informações complexas de redes sociais.

\section{MÉTODO}

De acordo com Gil (2017), esta pesquisa pode ser classificada como exploratória, tendo como base um estudo de caso realizado no Instituto Federal de Minas Gerais (IFMG) com enfoque em ampliar os conhecimentos sobre o processo de análise dos dados de candidatos a auxílios socioeconômicos e, a partir deste aprofundamento, sugerir possibilidades de melhorias suportadas por recursos tecnológicos. O planejamento do estudo de caso contemplou as seguintes etapas: entendimento do contexto atual e formulação do problema, indicação de solução potencial, obtenção dos dados, aplicação da solução proposta e preparação de relatório conclusivo.

A compreensão da realidade do IFMG em relação à concessão de auxílios socioeconômicos permitiu a identificação de quatro problemas no processo de análise dos dados de candidatos participantes de editais internos. Isso foi possível a partir da realização de entrevistas não estruturadas com Assistentes Sociais que atuaram como responsáveis pela verificação das informações prestadas pelos candidatos e pela definição de quais seriam contemplados com o recebimento dos auxílios ofertados.

Em busca de um recurso que pudesse aprimorar o processo de análise, constatou-se, com base nos trabalhos correlatos indicados na seção anterior, que uma possível solução seria disponibilizar aos Assistentes Sociais a visualização das informações em formato de grafos, pois o formato textual não favorece a identificação de relacionamentos entre os dados, o que era necessário neste caso estudado.

Considerando os problemas identificados na primeira etapa desta pesquisa, a solução tecnológica implantada no IFMG - descrita em Melo et al. (2021) - e a autorização formal concedida pela instituição, as informações necessárias para o estudo foram obtidas com a execução de consultas SQL (Structured Query Language) no repositório dos dados de candidatos e editais promovidos pelo IFMG. Os resultados das consultas foram salvos em arquivos no formato CSV (Comma-separated Values). Os dados obtidos foram armazenados na ferramenta Neo4j, um banco de dados orientado a grafo com recursos aderentes às aplicações modernas que demandam intensa conexão entre os dados. O carregamento dos arquivos CSV no Neo4j foi feito na ferramenta Neo4j Browser com comandos da linguagem de consulta Cypher, utilizada em aplicações que requerem simplicidade na escrita de instruções e eficiência no processo de manipulação de informações. A construção das visualizações dos dados em grafos foi feita com a ferramenta Neo4j Bloom.

\section{RESULTADOS}

O grande volume de dados coletados pelo setor de Assistência Estudantil do IFMG, em conjunto com a sua apresentação no formato textual, dificulta a análise e não atende às demandas dos Assistentes Sociais. Torna-se complexo compreender as relações do candidato com os membros do seu grupo familiar, bem como recuperar as informações específicas de cada membro para fornecer subsídios aos Assistentes Sociais na distribuição dos auxílios socioeconômicos. A Figura 1 apresenta o único recurso atualmente disponível para acesso aos dados dos membros do grupo familiar do candidato: um formulário textual que exibe todas as informações cadastradas pelo candidato, inclusive aquelas que não influenciam no deferimento do auxílio pleiteado. 


\begin{tabular}{|c|c|c|c|c|}
\hline \multicolumn{5}{|c|}{ MEMBRO 1} \\
\hline \multicolumn{3}{|l|}{ NOME } & CPF & $\begin{array}{l}\text { DATA NASCIMENTO } \\
03 / 01 / 1976\end{array}$ \\
\hline \multicolumn{2}{|c|}{$\begin{array}{l}\text { SITUAÇÃO DO TRABALHO PRINCIPAL } \\
\text { Trabalhador informal/eventual }\end{array}$} & \begin{tabular}{|l} 
PROFISSÃO \\
Ajudante de obras
\end{tabular} & \multicolumn{2}{|c|}{$\begin{array}{l}\text { ESTADO CIVIL } \\
\text { Casado ou uniäo estável }\end{array}$} \\
\hline \multicolumn{2}{|c|}{$\begin{array}{l}\text { RESPONSÁVEL PELO GRUPO FAMILIAR } \\
\text { Sim }\end{array}$} & $\begin{array}{l}\text { GRAU DE PARENTESCO } \\
\text { Pai }\end{array}$ & \multicolumn{2}{|c|}{$\begin{array}{l}\text { ESCOLARIDADE } \\
\text { Superior incompleto }\end{array}$} \\
\hline $\begin{array}{l}\text { ESTUDA NO IF } \\
\text { Sim }\end{array}$ & N CADÚNICO & \begin{tabular}{|l} 
RENDA MENSAL BRUTA \\
R $\$ 1.100,00$
\end{tabular} & \multicolumn{2}{|c|}{$\begin{array}{l}\text { RENDA MENSAL BRUTA (OUTROS) } \\
\text { R } \$ 0,00\end{array}$} \\
\hline \multicolumn{5}{|c|}{ MEMBRO 2} \\
\hline \multicolumn{3}{|l|}{ NOME } & CPF & \begin{tabular}{|l} 
DATA NASCIMENTO \\
06/01/2018
\end{tabular} \\
\hline \multicolumn{2}{|c|}{$\begin{array}{l}\text { SITUAÇÃO DO TRABALHO PRINCIPAL } \\
\text { Não trabalha }\end{array}$} & \begin{tabular}{|l|} 
PROFISSÃO \\
Nenhuma
\end{tabular} & \multicolumn{2}{|c|}{$\begin{array}{l}\text { ESTADO CIVIL } \\
\text { Solteiro }\end{array}$} \\
\hline \multicolumn{2}{|c|}{$\begin{array}{l}\text { RESPONSÁVEL PELO GRUPO FAMILIAR } \\
\text { Não }\end{array}$} & $\begin{array}{l}\text { GRAU DE PARENTESCO } \\
\text { Irmã(o) }\end{array}$ & \multicolumn{2}{|c|}{$\begin{array}{l}\text { ESCOLARIDADE } \\
\text { Ainda não frequenta a escola }\end{array}$} \\
\hline $\begin{array}{l}\text { ESTUDA NO IF } \\
\text { Não }\end{array}$ & N CADÚNICO & $\begin{array}{l}\text { RENDA MENSAL BRUTA } \\
\mathrm{R} \$ 0,00\end{array}$ & \multicolumn{2}{|c|}{$\begin{array}{l}\text { RENDA MENSAL BRUTA (OUTROS) } \\
\text { R } \$ 0,00\end{array}$} \\
\hline \multicolumn{5}{|c|}{ MEMBRO 3} \\
\hline \multicolumn{3}{|l|}{ NOME } & CPF & \begin{tabular}{|l} 
DATA NASCIMENTO \\
$01 / 02 / 1976$
\end{tabular} \\
\hline \multicolumn{2}{|c|}{$\begin{array}{l}\text { SITUAÇÃO DO TRABALHO PRINCIPAL } \\
\text { Não trabalha }\end{array}$} & \begin{tabular}{|l} 
PROFISSÃO \\
Do lar
\end{tabular} & \multicolumn{2}{|c|}{$\begin{array}{l}\text { ESTADO CIVIL } \\
\text { Casado ou união estável }\end{array}$} \\
\hline \multicolumn{2}{|c|}{$\begin{array}{l}\text { RESPONSÁVEL PELO GRUPO FAMILIAR } \\
\text { Não }\end{array}$} & $\begin{array}{l}\text { GRAU DE PARENTESCO } \\
\text { Mãe }\end{array}$ & \multicolumn{2}{|c|}{$\begin{array}{l}\text { ESCOLARIDADE } \\
\text { Fundamental incompleto }\end{array}$} \\
\hline $\begin{array}{l}\text { ESTUDA NO IF } \\
\text { Não }\end{array}$ & N CADÚNICO & $\begin{array}{l}\text { RENDA MENSAL BRUTA } \\
R \$ 0,00\end{array}$ & \multicolumn{2}{|c|}{$\begin{array}{l}\text { RENDA MENSAL BRUTA (OUTROS) } \\
\mathrm{R} \$ 0,00\end{array}$} \\
\hline
\end{tabular}

Figura 1. Dados dos membros do grupo familiar de um candidato. Fonte:Elaborado pelos autores (2021).

O entendimento sobre a composição do grupo familiar do candidato é importante para que o Assistente Social tenha uma noção mais aproximada da realidade do estudante e possa fazer a indicação daquele auxílio que terá impacto positivo na sua permanência na instituição. A Figura 2 apresenta as informações dos membros do grupo familiar no formato de grafo, para que o Assistente Social tenha uma visão mais direta das relações familiares, bem como acesso facilitado aos dados de cada membro ao usar o recurso de inspecionar do Neo4j. É possível notar que o Assistente Social pode buscar as relações de determinado candidato pelo CPF e, a partir da seleção de qualquer nó, visualizar seus dados específicos (como, neste exemplo, onde a situação de trabalho e a renda do familiar são exibidas). Ressalta-se que o nome do candidato foi alterado em função do sigilo dos dados.

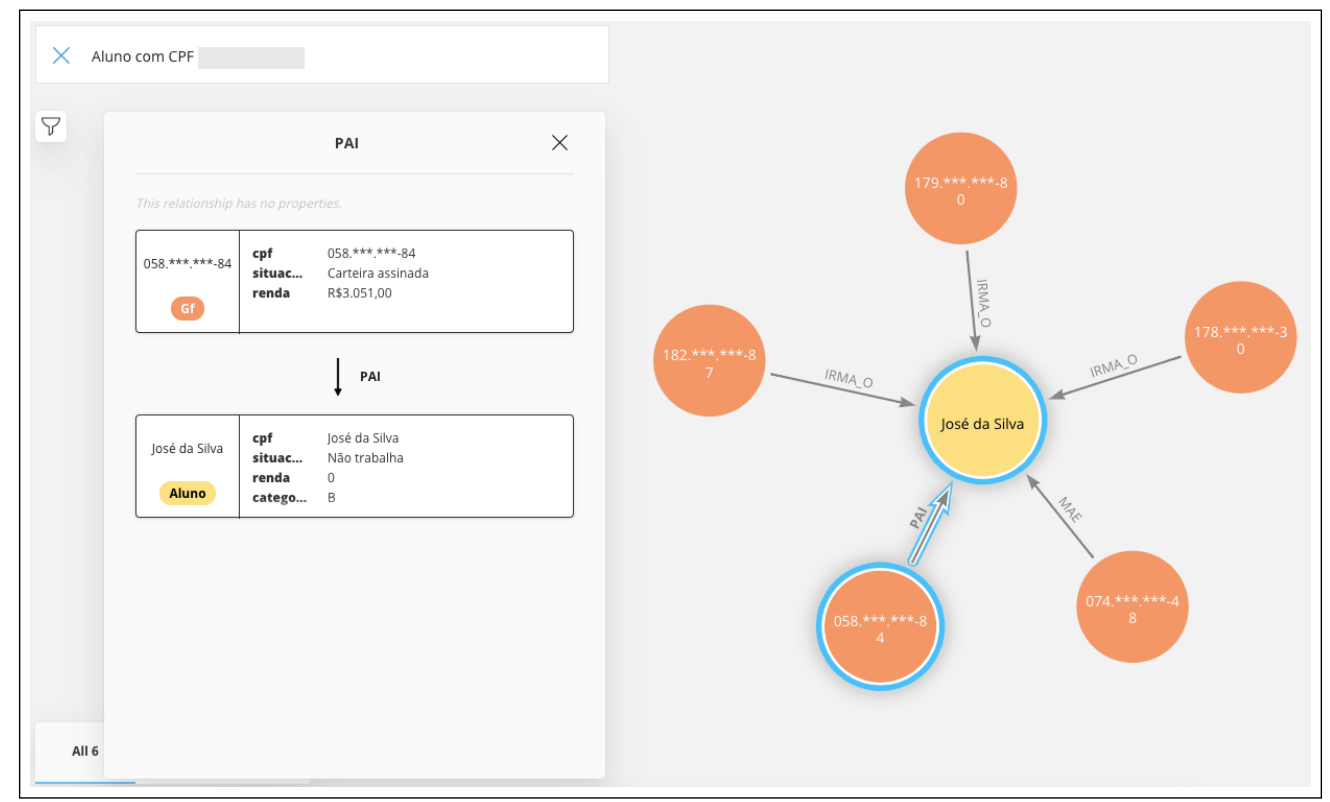

Figura 2. Visualização, em grafo, dos membros do grupo familiar de um candidato. Fonte:Elaborado pelos autores (2021).

Outra demanda identificada nos levantamentos realizados originou a elaboração do grafo apresentado na Figura 3, no qual o Assistente Social consegue visualizar a relação do candidato com os veículos de sua propriedade ou de membros do seu grupo familiar. Os dados de cada veículo também são, atualmente, disponibilizados apenas no formato textual, o que ocasiona dificuldades no processo de análise em função do volume de informações. O 
Neo4j Bloom permite a formatação dos nós com cores variadas de acordo com condições pré-determinadas. Neste exemplo, os veículos de propriedade do candidato são exibidos na cor verde, enquanto aqueles dos membros da família são apresentados na cor roxa. Destaca-se o fato de as informações de cada nó serem exibidas em uma janela específica, ao lado do grafo, quando requerido pelo usuário.

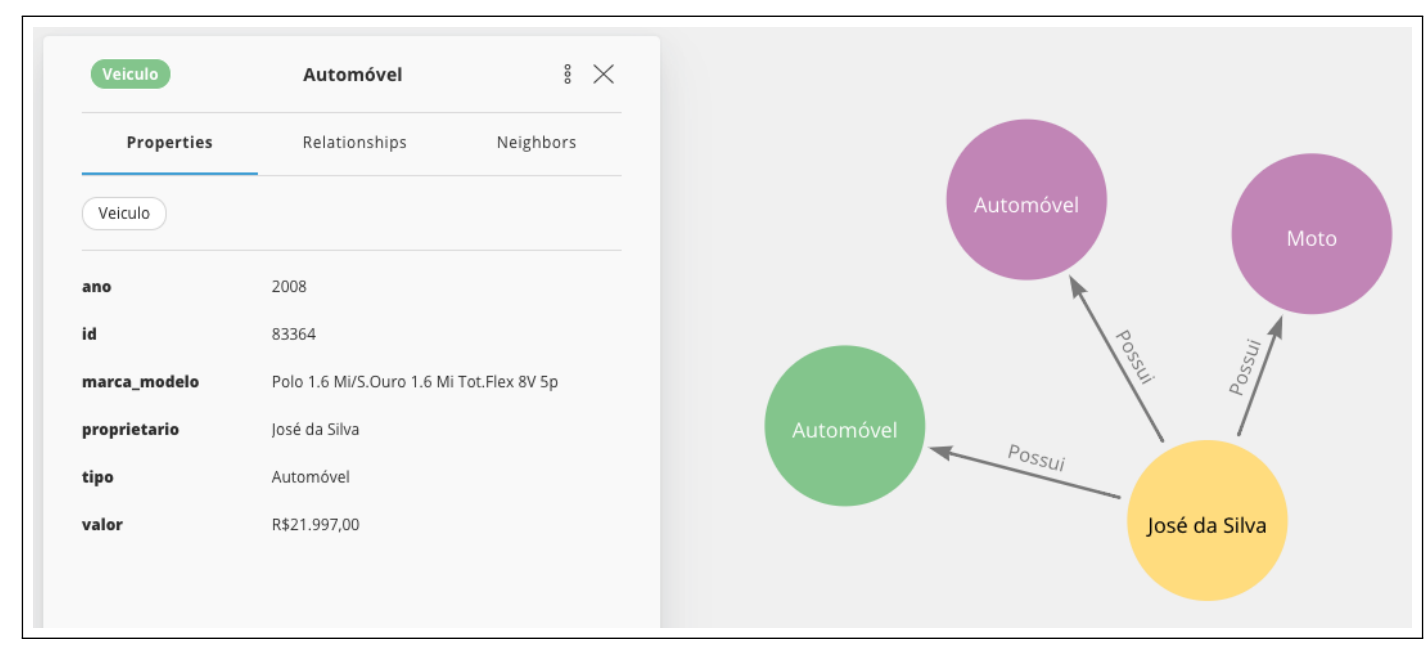

Figura 3. Visualização, em grafo, dos veículos atribuídos a um candidato. Fonte:Elaborado pelos autores (2021).

A participação do candidato em outros editais de concessão de auxílios socioeconômicos também é analisada pelos Assistentes Sociais durante o processo de seleção. É relevante saber, por exemplo, se o candidato foi aprovado em outras seleções, se consta como excedente em algum edital, se já recebeu auxílio ou se alguma solicitação já foi indeferida. A Figura 4 apresenta um grafo contendo o relacionamento do aluno com todos os editais em que ele participou. O esquema de cores do Neo4j Bloom auxilia na identificação visual do resultado do edital (verde para solicitações deferidas e vermelho para indeferidas), assim como a janela de inspeção apresentando os dados da solicitação selecionada, otimizam a análise pelo Assistente Social.

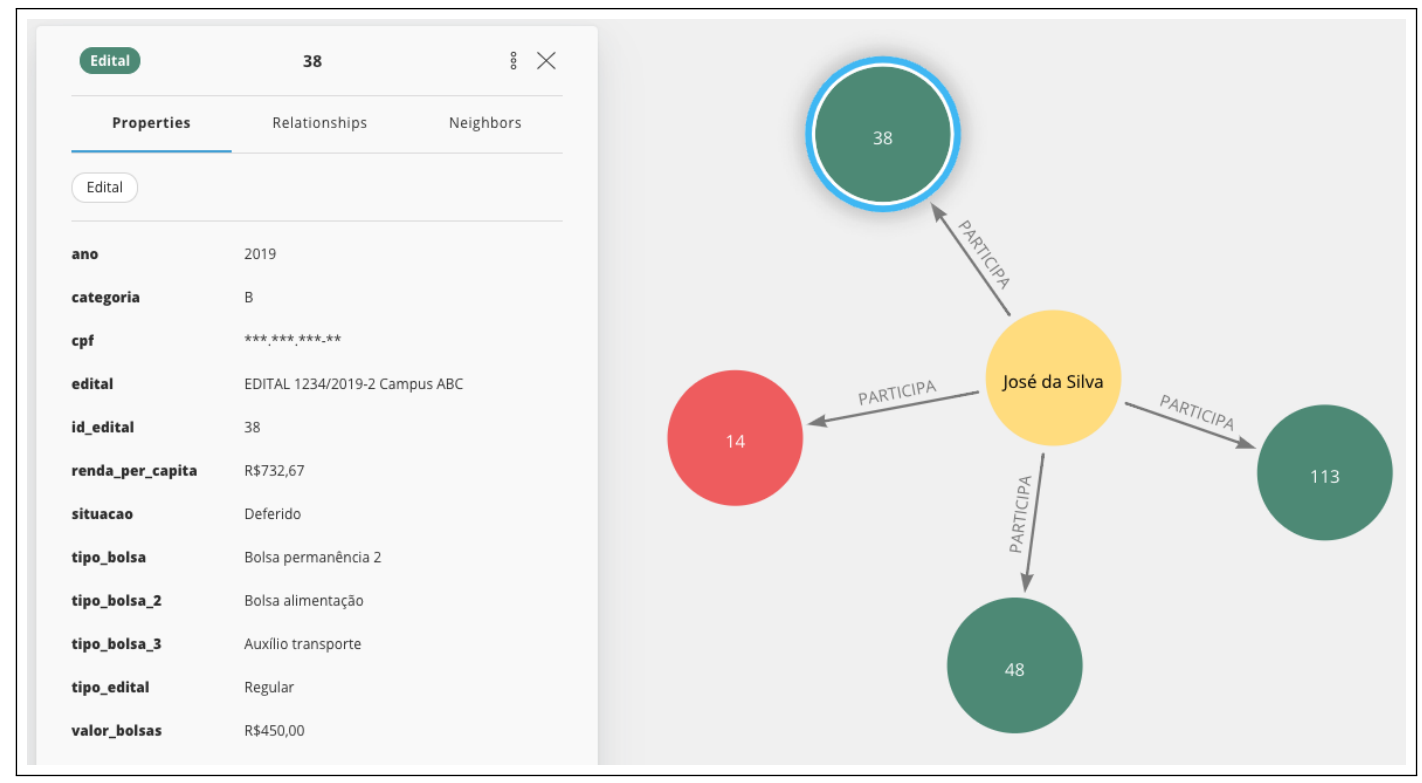

Figura 4. Visualização, em grafo, dos editais em que o candidato participa. Fonte:Elaborado pelos autores (2021).

A última visualização elaborada (Figura 5) exibe um grafo contendo todas as inscrições confirmadas em um edital, organizadas por cor e localização, de acordo com o tipo de auxílio pleiteado por cada candidato. Os itens marcados na cor azul indicam solicitações do auxílio "Bolsa Permanência I (R400, 00)j, itensempretodoauxlio1BolsaPermannciaII (R300, itens em amarelo do auxílio "Bolsa Permanência III (R200, 00)j, itensemlaranjadoauxlioıBolsaPermannciaIV (R100,00)" e itens em violeta do auxílio "Alimentação". Esta informação é útil para o Assistente Social obter subsídios para realizar a distribuição dos recursos da forma mais adequada possível, considerando as demandas apresentadas pelos candidatos. Por exemplo, se o número de requerimentos entre os tipos de auxílio estiver muito desbalanceado, pode ocorrer uma realocação dos recursos para que mais candidatos sejam atendidos. 


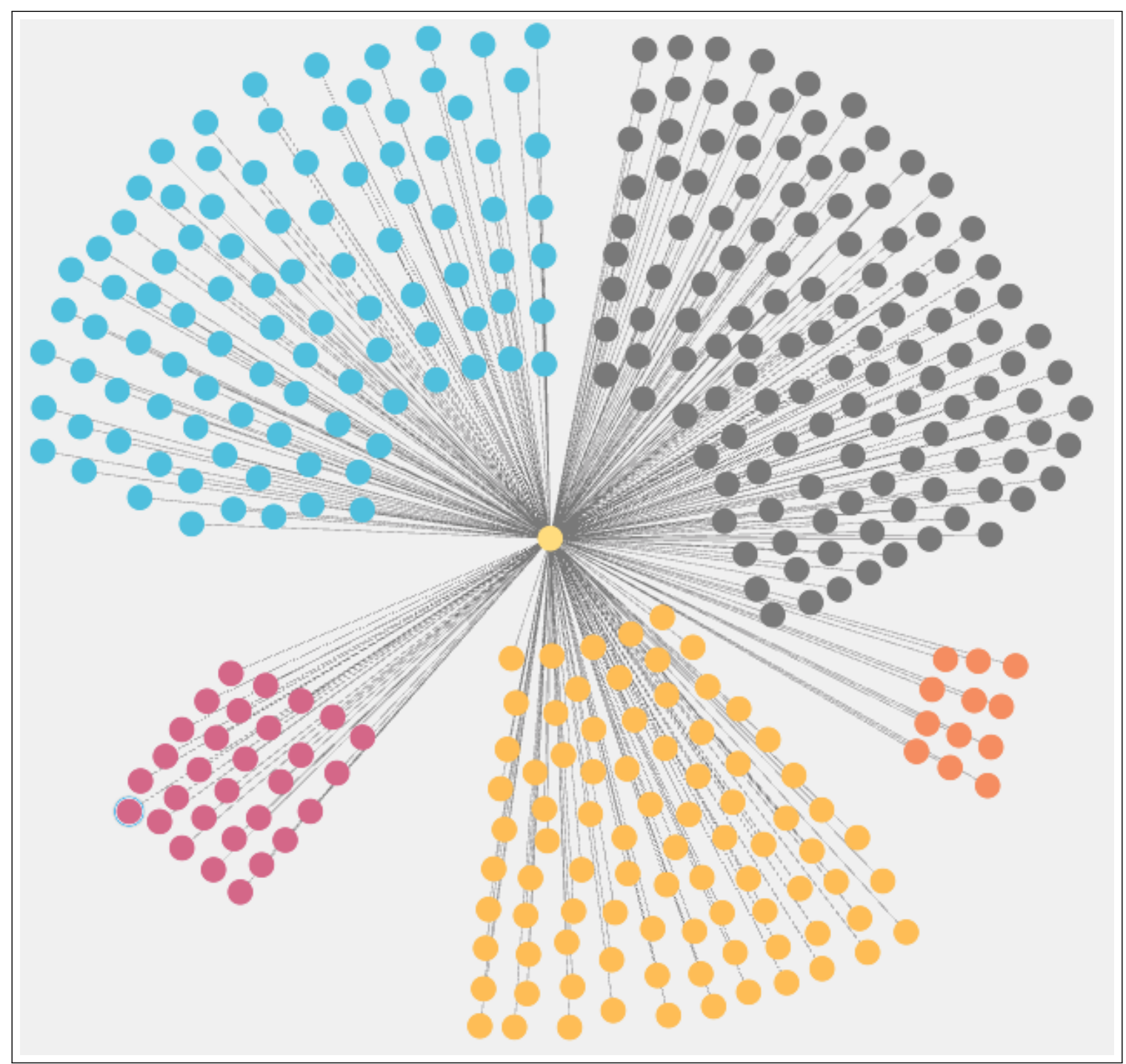

Figura 5. Visualização, em grafo, dos diferentes auxílios solicitados em um edital. Fonte:Elaborado pelos autores (2021).

Por fim, nota-se que a visualização dos dados como imagens tende a favorecer o entendimento de cada situação de maneira mais completa do que apenas a partir da análise dos dados em formato textual.

\section{CONCLUSÕES}

Apresentou-se, neste trabalho, como a visualização de informações em grafos pode ser explorada para otimizar tarefas que já vêm sendo executadas com o apoio de um sistema informatizado de gestão. Se, por um lado, este sistema auxilia no gerenciamento dos dados dos candidatos a auxílios socioeconômicos, por outro, ainda não oferece aos usuários - Assistentes Sociais - um formato que traduza de forma mais legível os diversos relacionamentos existentes entre essas informações, assim como indicado por Matsuba e Mettedi (2021). Como o usuário dispõe de um grande volume de dados para analisar e utilizar, é fundamental que estes realmente colaborem para que a decisão seja a mais coerente possível com cada situação. Esta questão se torna ainda mais pertinente ao considerarmos que a não atribuição de auxílios (ou atribuição de auxílios insuficientes) para aqueles candidatos com maior vulnerabilidade pode ocasionar sua não permanência na instituição e o consequente abandono dos estudos.

Exibir os dados demandados pelo processo de análise dos candidatos a auxílios socioeconômicos em um formato mais amigável e de fácil entendimento pode trazer resultados consideráveis na rotina de trabalho dos Assistentes Sociais do Instituto Federal de Minas Gerais. Não somente a qualidade das decisões pode ser melhorada com a agregação do recurso de visualização de informações em grafos de conhecimento, mas também o tempo gasto nas análises pode ser otimizado.

Este artigo considerou apenas a realidade específica do IFMG, limitando, assim, possíveis generalizações destas conclusões sobre os pontos positivos do recurso implementado. As visualizações construídas neste estudo dependem da disponibilidade de dados que, eventualmente, não são coletados nem estão disponíveis em outras 
instituições ou setores de Assistência Estudantil. Espera-se implementar e disponibilizar em ambiente de produção todas as visualizações de informações em grafos apresentadas neste artigo, bem como estudar o impacto real deste recurso no trabalho dos Assistentes Sociais do IFMG em relação aos dois aspectos citados nesta conclusão: a qualidade das decisões tomadas sobre atribuição de auxílios, e o tempo gasto para condução das análises. 


\section{REFERÊNCIAS}

Andrade, A. M. J., \& Teixeira, M. A. (2017). Áreas da política de assistência estudantil: relação com desempenho acadêmico, permanência e desenvolvimento psicossocial de universitários. Revista da Avaliação da Educação Superior, 22(2). Recuperado de https://www.scielo.br/j/aval/ a/wqhZ5Km7XCbNbPTRwQrjP6x/?lang=pt

Associação Nacional dos Dirigentes das Instituições Federais de Ensino Superior [ANDIFES. (2019). V pesquisa do perfil socioeconómico e cultural dos estudantes de graduação das instituições federais de ensino superior brasileiras. Brasília, DF: ANDIFES. Recuperado de https://www.andifes.org.br/wp-content/uploads/2019/05/ V-Pesquisa-Nacional-de-Perfil-Socioeconomico-e-Cultural -dos-as-Graduandos-as-das-IFES-2018.pdf

Bezerra, M., Medeiros, G., \& Oliveira, M. (2014). Visualização de informação de opiniões online sobre restaurantes: uso de técnicas orientadas à visualização de grafos. Revista Eletrônica de Sistemas de Informação, 6. Recuperado de https://archhealthinvestigation.com.br/ArcHI/article/ view $/ 2661$

Bezerra, M., Medeiros, G., \& Oliveira, M. (2016). Dpibicjr oo1 - aplicativo de monitoramento e tomada de decisão utilizando rede social e rede de grafos: estudo de caso zika vírus. Archives of Health Investigation, 6. Recuperado de https://archhealthinvestigation.com.br/ArcHI/article/ view $/ 2661$

Boaventura Netto, P. O., \& Jurkiewicz, S. (2017). Grafos: introdução e prática. São Paulo: Blucher.

Braga, M. C. M. (2017). A política de assistência estudantil na Universidade Federal do Rio Grande do Sul: um estudo comparativo da eficácia na aplicação da verba do Programa Nacional de Assistência Estudantil através do desempenho acadêmico dos estudantes (Dissertação de mestrado, Universidade Federal do Rio Grande do Sul, Porto Alegre, RS, Brasil). Recuperado de https://lume.ufrgs.br/ handle/10183/168648

Camargo, A. M. M., \& Araújo, I. M. (2018). Expansão e interiorização das universidades federais no período de 2003 a 2014: perspectivas governamentais em debate. Acta Scientiarum Education, 40(1).

Chaves, J., Farias, M., Nunes, A., \& Araújo, W. (2021). Análise e melhoria processos informacionais na assistência estudantil. Revista FSA, 18(2), 162-182. Recuperado de http://www4.unifsa.com.br/revista/index.php/fsa/ article/view/2225

Costa, C. C. S. (2018). Modelagem de algoritmos de distribuição espacial de grafos (Dissertação de mestrado, SINAI/CIMATEC, Salvador, BA, Brasil). Recuperado de http://repositoriosenaiba.fieb.org.br/handle/fieb/890

Decreto n. 7.234, de 19 de julho de 2010. (2010). Dispõe sobre o Programa Nacional de Assistência Estudantil (PNAES). Brasília, DF. Recuperado de http://www.planalto.gov.br/ ccivil_03/_ato2007-2010/2010/decreto/d7234.htm

Detlor, B. (2010). Information management. Informação e Informação, 30. doi: 10.1016/j.ijinfomgt.2009.12.001

Eloi, S. S. S., Silva, F. C., Silveira, S. F. R., \& Baeta, O. V. (2019). O programa nacional de assistência estudantil-pnaes na universidade federal de viçosa (ufv): uma avaliação na percepção dos beneficiários. Revista Gestão Universitária na América Latina, 12(3). Recuperado de https://periodicos.ufsc.br/index.php/gual/article/view/

\section{$1983-4535.2019 v 12 n 3 p 106$}

Gil, A. C. (2017). Como elaborar projetos de pesquisa. São Paulo: Atlas.

Instituto Federal de Minas Gerais [IFMG]. (2020). Resolução n. 9 de 03 de julho de 2020. Dispõe sobre a aprovação da Política de Assisténcia Estudantil no âmbito do IFMG. Belo Horizonte, MG: IFMG. Recuperado de https://www2.ifmg.edu.br/portal/sobre-o-ifmg/ conselho-superior/resolucoes /2020-3/resolucao-no-009 -2020-aprovacao-da-politica-de-assistencia-estudantil-no -ambito-do-ifmg-e-revogacao-da-resolucao-no-3-2019/view

Lucchesi, C. L. (1979). Introdução à teoria dos grafos. São Paulo: IMPA.

Machado, G. C., Oliveira, C. A., \& Freitas, T. A. (2017). Avaliação do impacto dos benefícios pnaes sobre o desempenho acadêmico: o caso da universidade federal do rio grande. In 3 simpósio avaliação da educação superior. Florianópolis, $\mathrm{SC}$.

Mariano, E. B. (2020). Gestão da informação educacional: um estudo de caso sobre a qualificação dos dados e informações no IFSC (Dissertação de mestrado, Instituto Federal de Santa Catarina, Florianópolis, SC, Brasil). Recuperado de https://repositorio.ifsc.edu.br/handle/123456789/1589

Martins, P. F. M., Araújo Júnior, C. A. M., \& Rodrigues, J. A. (2018). Programa de promoção à permanência: perfil dos discentes do instituto federal de ciência e tecnologia de brasília. HOLOS, 3, 379-384. Recuperado de https://www2 .ifrn.edu.br/ojs/index.php/HOLOS/article/view/4044

Martins, P. F. M., Araújo Júnior, C. A. M., \& Rodrigues, J. A. (2019). Orçamento público para financiamento da assistência estudantil no ensino superior sob a perspectiva do direito humano fundamental à educação. Revista Argumentos, 16(2), 138-164. Recuperado de https://www.periodicos .unimontes.br/index.php/argumentos/article/view/214

Matsuba, D. S., \& Mettedi, A. P. (2021). Visualização de dados para extração de conhecimentos: um estudo de caso. AtoZ: novas práticas em informação e conhecimento, 10(2), 66-77. doi: 10.5380/atoz.v10i2.79184

Melo, E. C., Silva, G., \& Silva, P. C. L. (2021). Informatização do processo de seleção de bolsistas de assistência estudantil: a experiência do ifmg. Research, Society and Development, 10(1). Recuperado de https://rsdjournal.org/ index.php/rsd/article/view/11778

Ministério da Educação. (2012). Análise sobre a expansão das Universidades Federais 2003 a 2012. Brasília, DF. Recuperado de dehttp://portal.mec.gov.br/docman/ janeiro-2013-pdf/12386-analise-expansao-universidade -federais-2003-2012-pdf

Ministério da Educação [MEC]. (2021). Instituições da rede federal. Brasília, DF: MEC. Recuperado de http:// portal.mec.gov.br/rede-federal-inicial/instituicoes

Moraes, N. J., \& Spessatto, M. B. (2020). Ensino superior público no brasil: o percurso histórico e o papel da assistência estudantil no acesso e permanência dos estudantes. ScientiaTec, 7(4). Recuperado de https://periodicos.ifrs.edu.br/ index.php/ScientiaTec/article/view/4746

Ramos, D. B. (2016). Uma ferramenta baseada em grafo para identificação e visualização de trilhas de aprendizagem (Dissertação de mestrado, Universidade Federal do Amazonas, Manaus, AM, Brasil). Recuperado de https:// tede.ufam.edu.br/handle/tede/5031

Saccaro, A., França, M. A., \& Jacinto, P. A. (2016). 
Retenção e evasão no ensino superior brasileiro: uma análise dos efeitos da bolsa permanência do pnaes. In Encontro nacional de economia-anpec 44. Recuperado de https://www.anpec.org.br/encontro/2016/submissao/ files_I/i12-707be73e05304f27eb9f18ae5d235c9d.pdf

Sales, E. C. S. S. (2018). Reestruturação e Expansão das Universidades Federais: Avaliação dos Resultados do Programa Reuni sob a Perspectiva de Políticas Públicas para a Educação Superior (Dissertação de mestrado, Universidade Federal de á, Goiânia, GO, Brasil). Recuperado de https://repositorio.bc.ufg.br/tede/handle/tede/9229\#: :text=O\%20Programa $\% 20$ de $\% 20$ Apoio $\% 20$ a,dado $\% 20$ o $\%$ 20baixo\%20\% C3\%ADndice\%20de

Santos, C., Abrantes, P. P. M., \& Zonta, R. (2021). Limitações orçamentárias: desafios à assistência estudantil da unb em tempos de pandemia. Cadernos Cajuina, 6(3). Recuperado de https://cadernoscajuina.pro.br/revistas/index.php/ cadcajuina/article/view/503

Santos, E. H. M. (2020). As configurações da Assistência Estudantil no Brasil: o cenário do PNAES (Dissertação de mestrado, Universidade Federal de São Paulo, Brasil). Recuperado de https://repositorio.unifesp.br/handle/11600/ 60792

Sanz, I. J., \& Lopez, M. A. (2018). Um sistema de detecção de ameaças distribuídas de rede baseado em aprendizagem por grafos. In Simpósio brasileiro de redes de computadores e sistemas distribuídos (sbrc). Porto Alegre. Recuperado de https://sol.sbc.org.br/index.php/sbrc/article/view/2487

Silva, L. B., \& Costa, N. C. D. (2018). Acesso e permanência em desproporção: as insuficiências do programa nacional de assistência estudantil. Agenda Política, 6(1), 166-192. Recuperado de https://www.agendapolitica.ufscar.br/index .php/agendapolitica/article/view/208

Silva, T. E., \& Tomaél, M. I. (2007). A gestão da informação nas organizações. Informação e Informação, 12(2). Recuperado de https://www.uel.br/revistas/uel/index.php/ informacao/article/view/1806/0

Silva, T. E., \& Tomaél, M. I. (2016). Fundamentações teóricas para a criação de um modelo de gestão da informação para o contexto da avaliação de cursos de graduação. Biblionline, 12(2). Recuperado de https://periodicos.ufpb.br/ index.php/biblio/article/view/27890

Silva Júnior, J. M. (2020). Eficácia dos programas de assistência estudantil: experiência da UNEMAT (Dissertação de mestrado, Universidade Federal do Ceará, Fortaleza, CE, Brasil). Recuperado de http://www.repositorio.ufc.br/ handle/riufc/54102

Szwrcfiter, J. L. (1984). Grafos e algoritmos computacionais. Rio de Janeiro: Campus.

Terenciano, F., Salião, A., \& Pedro, Z. (2018). Uso da gestão da informação e do conhecimento na avaliação da política pública de assistência estudantil. Regae, 7(14), 49-62. Recuperado de https://periodicos.ufsm.br/regae/ article/view/29803

Como citar este artigo (APA):

Melo, E. C., Bax, M. P., \& Marques, Y. B. (2022). Visualização dos dados de candidatos a auxílios socioeconômicos em grafos: estudo de caso no Instituto Federal de Minas Gerais (IFMG). AtoZ: novas práticas em informação e conhecimento, 11,1 - 11. Recuperado de: http://dx.doi.org/10.5380/atoz.v11.82607 


\section{NOTAS DA OBRA E CONFORMIDADE COM A CIÊNCIA ABERTA}

\section{CONTRIBUIÇÃO DE AUTORIA}

\begin{tabular}{|l|c|c|c|}
\hline $\begin{array}{l}\text { Papéis } \\
\text { e contribuições }\end{array}$ & $\begin{array}{c}\text { Eduardo } \\
\text { Cardoso } \\
\text { Melo }\end{array}$ & $\begin{array}{c}\text { Marcello } \\
\text { Peixoto Bax }\end{array}$ & $\begin{array}{c}\text { Yuri Bento } \\
\text { Marques }\end{array}$ \\
\hline Concepção do manuscrito & $\mathrm{X}$ & $\mathrm{X}$ & $\mathrm{X}$ \\
\hline Escrita do manuscrito & $\mathrm{X}$ & $\mathrm{X}$ & $\mathrm{X}$ \\
\hline Metodologia & $\mathrm{X}$ & $\mathrm{X}$ & \\
\hline Curadoria dos dados & $\mathrm{X}$ & $\mathrm{X}$ & \\
\hline Discussão dos resultados & $\mathrm{X}$ & $\mathrm{X}$ & $\mathrm{X}$ \\
\hline Análise dos dados & $\mathrm{X}$ & $\mathrm{X}$ & $\mathrm{X}$ \\
\hline
\end{tabular}

\section{Disponibilidade de Dados Científicos da Pesquisa}

Os conteúdos subjacentes ao texto da pesquisa estão disponíveis sob demanda dos pareceristas.

\section{EQUIPE EDITORIAL}

\section{Editora/Editor Chefe}

Maria do Carmo Duarte Freitas (https://orcid.org/0000-0002-7046-6020)

\section{Editora/Editor Associada/Associado}

Paula Carina de Araújo (https://orcid.org/0000-0003-4608-752X)

\section{Editora/Editor de Texto Responsável}

Fabiane Führ (https://orcid.org/0000-0002-3723-050X)

Nicholle Ferreira Murmel Liali (https://orcid.org/0000-0002-1086-908X)

\section{Editora/Editor de Layout}

Karolayne Costa Rodrigues de Lima (https://orcid.org/0000-0002-6311-8482) 